\title{
A Magnet Retained Facial Prosthesis for A Cancer Survivor- A Report From A Tertiary Cancer Centre.
}

\author{
Pramod Sankar. $S^{1}$, Rajitha A.V ${ }^{2}$ \\ ${ }^{1}$ (Associate Professor, Dept Of Dentistry\&Rehabilitation, Malabar Cancer Centre,Thalassery) \\ ${ }^{2}$ (Assistant Dental Surgeon, TH Hospital, Nileshwar)
}

\begin{abstract}
Extra-oral prosthesis improves the appearance and enhances confidence of the patient. Large facial defects involving lower half of the face are very difficult to treat prosthetically because of the mobility of the soft tissue margins, opening and closing movement of the mandible and poor retentive features of the defect. This article features a cancer survivor for whom a facial prosthesis was fabricated to obturate the defect that resulted from a free flap failure. An acrylic scaffold provided the base for medical grade silicone, which restored the facial form. The extra-oral prosthesis was retained with the help of magnets attached to maxillary and mandibular intra-oral prosthesis. Eventhough the prosthesis didn't restore the function, patient accepted the prosthesis well as it helped him for public appearances.
\end{abstract}

Keywords: Facial prosthesis, Silicone, magnets.

\section{Introduction}

Large facial deformities can result from treatment for tumors, trauma, burns, and congenital anomalies. Extra-oral prosthesis are planned for patients where a reconstructive surgical option is not viable either due to some co-morbidities or for aesthetic reasons. ${ }^{1,2}$ These prosthesis provides excellent results if they rest on static tissues. Auricular prosthesis, nasal prosthesis and orbital prosthesis functions well in that perspective. Facial prosthesis always pose a challenge to the prosthodontist as the defect involves the middle and lower third of the face which is highly mobile, during facial expressions or when opening the mouth. In such instances an accurate marginal seal is impossible. This clinical report describes the maxillofacial prosthetic management of a patient who underwent multiple surgical resections for squamous cell carcinoma of retromolar trigone.

\section{Clinical Report}

A 49 year old male patient underwent surgery followed by adjuvant radiotherapy for moderately differentiated squamous cell carcinoma of right retromolar trigone.(Stage IV A- $\mathrm{T}_{4 \mathrm{a}} \mathrm{N}_{1} \mathrm{M}_{0}$ ) A full thickness wide excision with right side hemimandibulectomy, right modified radical neck dissection and reconstruction with folded Pectoralis Major Myocutaneous (PMMC) flap was done and 60Gy/30\# adjuvant radiation dose was delivered. He developed a recurrence at right gingivo-buccal sulcus one year later for which he underwent infrastructure maxilectomy and right Latissimus Dorsal (LD) flap reconstruction. But the flaps failed resulting in a surgical defect as shown in figure 1.The patient was referred to prosthetic department to close the defect until a Free Radial Artery Forearm (FRAFF) flap was planned later.

\subsection{Extra-oral defect}

The defect involved right commisure and right cheek extending posteriorly upto $2.5 \mathrm{~cm}$ from tragus. Superiorly the defect was approximating the ala-tragal line; inferiorly it was close to the lower border of the mandible and was lined by the residual scar tissue. (Fig 1) The mandible was deviated to the right resulting in facial asymmetry. But the remaining dentition on left side guided the mandible into occlusion.

\subsection{Intra-oral findings}

All the maxillary anterior teeth, maxillary left second premolar, fist and second molar, mandibular left canine, left premolars and second molar were present. Right maxillary alveolus distal to 13 was absent. The right hemimanibulectomy resulted in mandibular edentulous ridge extending only upto canine region. All the teeth present were periodontally stable and free of any caries. The tongue was positioned laterally to the defect side with minimal movement. The patient couldn't swallow and was put on Nasogastric Tube (NGT) feeding. The speech was also not clear due to restricted tongue movement and open space.

\subsection{The prosthesis design.}

The prosthesis was aimed at obturating the defect with an acceptable cosmetic camouflage. He was made aware of the functional limitations of the prosthesis with regard to speech, mastication, and deglutition. The extra-oral prosthesis which restored the lips, facial form and contour were attached to the two intra-oral 
prosthesis by means of magnets. The intra-oral prosthesis were processed in heat cured PMMA and was retained with wrought wire clasps. The extra-oral prosthesis had an acrylic scaffold to which the silicone was bonded. Retentive holes and the primer provided necessary adhesion.

\subsection{Procedure}

Intra-oral impressions were made using irreversible hydrocolloid (Algitex, DPI, India) as per standard procedures. Cast were prepared in Type III Dental stone.( Gold stone, Asian Chemicals, India) (Fig 2). Maxillary and mandibular intra-oral prosthesis were fabricated in poly methyl methacrylate (PMMA) resin with suitable retentive clasps engaging the teeth present. Four mandibular incisors were replaced in the prosthesis to provide adequate lip support. Mandibular prosthesis had an L-shaped extension that closely approximated the inferior and posterior border of the defect. The prosthesis were inserted and checked for retention. The patient was instructed to wear the prosthesis for one week and evaluated for any discomfort. PMMA resin was trimmed at three different locations in the external surface of both the prosthesis to accommodate neodymuium samarium magnets. Magnets were attached with the help of auto-polymerising PMMA resin and a thin layer of resin covered the superficial surfaces. (Fig 2)

An extra-oral impression was made with the prosthesis in position using irreversible hydrocolloid and cast poured in Type III dental stone. A scaffold was prepared over the cast using auto-polymerising acrylic resin, with new magnets attached to the corresponding sites so that after processing the scaffold retained those magnets. It was ensured that the magnets were positioned with opposite poles coming in contact. The scaffold was perforated to provide additional retention for silicone elastomer. The scaffold was tried for its fit and retention with intra-oral prosthesis. (Fig 3)

Another extra-oral impression with the scaffold in situ was made. The cast was poured, with the scaffold in the impression, in Type IV dental stone. (Die stone, Maarc, Shiva Products, India). Modelling Wax (Hindustan dental products India) was used to carve the lips contours and facial form.(Fig 4). The wax framework was tried several times and necessary corrections were done. After final confirmation of the outline, contour and form, the surface pattern was established.

Medical grade silicone elastomer (P\&O International, India) was subjected to intrinsic staining ${ }^{5}$ with standard colorants to match the skin tone by a trial and error method. The selected shades were marked and recorded. The wax pattern was invested, mould prepared and processed in silicone elastomer with selected intrinsic stains according to standard processing techniques. ${ }^{3,4}$ The silicone prosthesis was retrieved; excess trimmed and try-in done. (Fig 5) The colour tone was further improved with extrinsic stains. The surface was subjected to sodium bicarbonate treat to provide a matted texture.

Prosthesis was inserted and checked for fit retention and esthetics. Patient was instructed regarding insertion, removal and cleaning of the prosthesis. (Fig 6)

\section{Discussion}

This prosthesis design was complicated in that it involved the commisure of the lip. Providing a marginal seal during mouth opening was impossible with a single prosthesis. If two separate prosthesis were planned, then the lower half prosthesis will be pushed laterally by the tongue which will further compromise the marginal seal. Considering the fact that the patient can't resort to normal swallowing and will be continued on NGT feeding until another surgery is planned, a single prosthesis was finalized to obturate the defect.

The extra-oral impression was made when the patient's mandible was in rest position. This was to avoid the muscle fatigue due to continous occlusal contact position. The scaffold and the magnets ensured that the mandible was in comfortable position during impression making.

PMMA resins and silicones are usually the material of choice for facial prosthesis. ${ }^{6-8}$ when compared to acrylic resin silicone can produce life-like, soft, light weight prosthesis. . But to incorporate some retentive features, an acrylic scaffold is necessary to back the silicone. In addition, acrylic scaffold needs to be primed with an agent that enhances adhesion with silicone. A two-component medical grade platinum silicone provided enough working time for mixing the intrinsic color and matching it with the records. The silicone was processed at room temperature for 48 hours. After final processing the prosthesis was stained with extrinsic stains and processed in microwave oven for 10 minutes. ${ }^{9}$

The final result after prosthetic treatment was acceptable from a cosmetic standpoint but did not improve the function. Drooling of saliva continued to be a problem for the patient. Further, the margins of the prosthesis were in constant contact with moisture, causing local irritation. However, the patient managed to wear the prosthesis for public appearances. The prosthesis enabled the patient to gain confidence and thus improved his quality of life.

Although surgical reconstruction of this type of facial defect may have resulted in completion of the oral seal, the cosmetic result would have been marginal because of difficulty in reconstructing the detailed 
contours of the lip, commissure and cheek areas. Continued research for methods and materials used in prosthetic reconstruction is needed for these patients for whom surgical reconstruction is not an option.

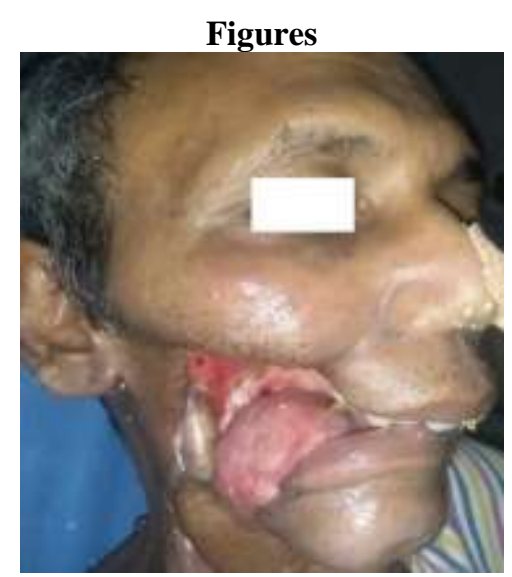

Figure 1- The extra-oral defect

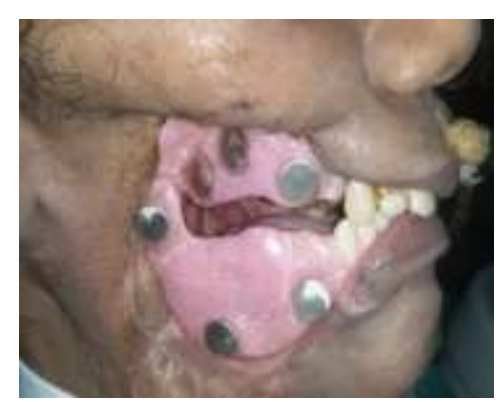

Figure 2- The intra-oral prosthesis with multiple magnets

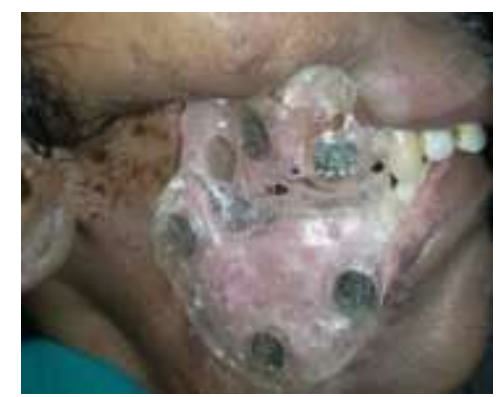

Figure 3- Magnet retained extra-oral scaffold for silicone prosthesis

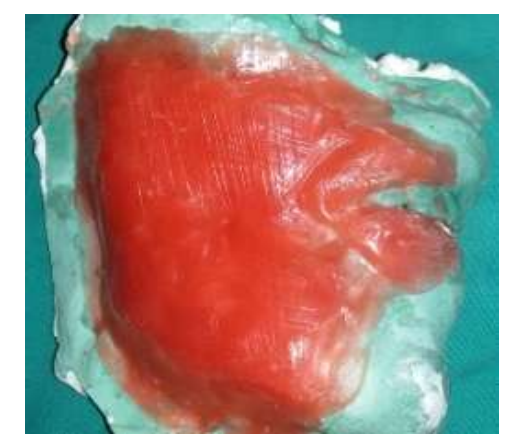

Figure 4- Wax form of the prosthesis over the scaffold 


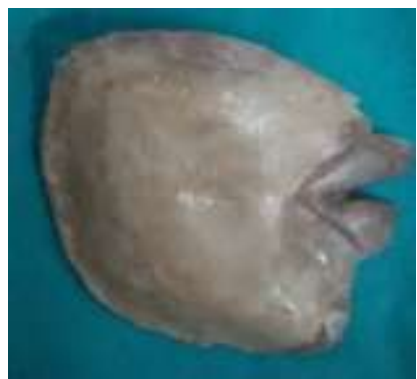

Figure 5- The silicone prosthesis

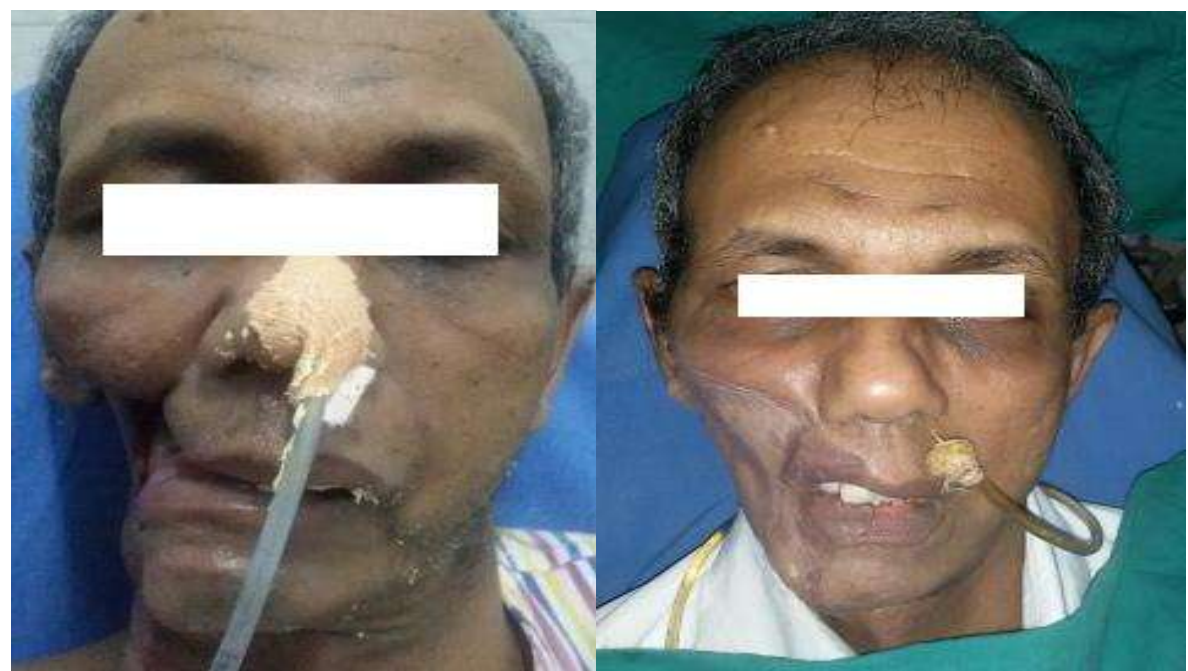

Figure 6 Patient with and without prosthesis

\section{Conclusion}

This clinical report describes the maxillofacial prosthetic management of a patient who underwent multiple surgical resections for squamous cell carcinoma of retromolar trigone

\section{References}

[1]. Beumer J, Curtis TA, Marunick MT. Maxillofacial Rehabilitation. Prosthodontic and Surgical Considerations. St. Louis: Medico Dental Media International,1996,377-85.

[2]. Stewart KL, Rudd KD, Kuebker WA: Other Forms of Removable Partial Denture. Clinical Removable Partial Prosthodontics. St. Louis, MO, Mosby, 1983,588-624.

[3]. Udagama A, Drane JB. Use of medical-grade methyl triacetoxy silane crosslinked silicone for facial prostheses. J Prosthet Dent 48,1982,86-8.

[4]. Farah JW, Robinson JC, Koran A, Craig RG, Hood JA. Properties of a modified cross-linked silicone for maxillofacial prostheses. J Oral Rehabil,14, 1987,599-605.

[5]. Thomas KF Color matching in: Prosthetic rehabilitation. Quintessence Publication Co. Ltd., London,1994,85

[6]. Mahajan Harsh, Gupta Kshiliz, Maxillofacial prosthetic material: A literature review J Orofac Res 2012,87-90.

[7]. Khindria SK, Bansal S, Kansal M, Maxillofacial prosthetic materials- Review Article J Indian Prosthodont Soc 2009,2-5.

[8]. Maller Udita S, Karthik KS, Maller Sudhakara V, Maxillofacial Prostheric Materials - Past \& Present Trends J Acquir Immune Defic Syndr 2010,26-30.

[9]. Huber H, Studer Stephan P, Materials and techniques in maxillofacial prosthodontic rehabilitation Oral Maxillofacial Surg Clin N Am 2002,73-93. 